\title{
ARNON AVRON
}

\section{Implication, Equivalence, and Negation}

\author{
Arnon Avron \\ School of Computer Science, \\ Tel Aviv University. \\ Tel Aviv 6997801, Israel. \\ E-mail: aa@cs.tau.ac.il
}

\begin{abstract}
A system $H C L_{\rightleftarrows}$ in the language of $\{\neg, \leftrightarrow\}$ is obtained by adding a single negation-less axiom schema to $H L L_{\rightrightarrows}$ (the standard Hilbert-type system for multiplicative linear logic without propositional constants), and changing $\rightarrow$ to $\leftrightarrow . H C L_{\rightleftarrows}$ is weakly, but not strongly, sound and complete for $\mathbf{C L}_{\rightleftarrows}$ (the $\{\neg, \leftrightarrow\}$-fragment of classical logic). By adding the Ex Falso rule to $H C L_{\rightrightarrows}$ we get a system with is strongly sound and complete for $\mathbf{C L}_{\rightleftarrows}$. It is shown that the use of a new rule cannot be replaced by the addition of axiom schemas. A simple semantics for which $H C L_{\rightleftarrows}$ itself is strongly sound and complete is given. It is also

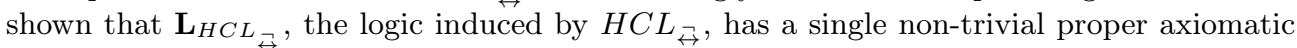
extension, that this extension and $\mathbf{C} \mathbf{L}_{\rightleftarrows}$ are the only proper extensions in the language of $\{\neg, \leftrightarrow\}$ of $\mathbf{L}_{H C L_{\rightleftarrows}}$, and that $\mathbf{L}_{H C L_{\rightleftarrows}}$ and its single axiomatic extension are the only logics in $\{\neg, \leftrightarrow\}$ which have a connective with the relevant deduction property, but are not equivalent to an axiomatic extension of $\mathbf{R}_{\rightrightarrows}$ (the intensional fragment of the relevant logic $\mathbf{R}$ ). Finally, we discuss the question whether $\mathbf{L}_{H C L_{\rightleftarrows}}$ can be taken as a paraconsistent logic.
\end{abstract}

Keywords: Implication, Semi-implication, Negation, Equivalence, Biconditional, Classical propositional logic, Deduction theorems, Paraconsistent Logics

For citation: Avron A. "Implication, Equivalence, and Negation", Logicheskie Issledovaniya / Logical Investigations, 2021, Vol. 27, No. 1, pp. 31-45. DOI: 10.21146/20741472-2021-27-1-31-45

\section{Introduction}

The relevant deduction property (RDP) for a binary connective $\rightarrow$ is a weak form of the classical-intuitionistic deduction theorem which has (somewhat implicitly) motivated the design of the intensional fragments $\left(\mathbf{R}_{\rightarrow}\right.$ and $\left.\mathbf{R}_{\neg}\right)$ of the relevance logic $\mathbf{R}$ (|Anderson, Belnap, 1975; Dunn, Restall, 2002 $).{ }^{1}$ In $\overrightarrow{\text { Avron, }}$ 2015 we showed that with one exception, in the pure language of $\{\rightarrow\} \rightarrow$ has in

\footnotetext{
${ }^{1}$ The RDP is also the key condition that should be satisfied by what is called in Avron, 2015 'semi-implication'. The other condition, included to avoid degenerate cases, is that not every case in which an implication holds is a case in which its converse also holds.
} 
a finitary logic $\mathbf{L}$ the RDP iff $\mathbf{L}$ has a strongly sound and complete Hilbert-type system which is an axiomatic extension (i.e. an extension by axiom schemas) of $H R_{\rightarrow}$ (the standard axiomatization of $\mathbf{R}_{\rightarrow}$ ). The only exception is a logic which, like $\mathbf{R}_{\rightarrow}$, is an axiomatic extension of $\mathbf{L} \mathbf{L}_{\rightarrow}$ (the purely implicational fragment of Linear Logic): $\mathbf{C L}_{\leftrightarrow}$, the pure equivalential fragment of classical logic (where the biconditional is denoted by $\rightarrow$ ).

The fact that $\leftrightarrow$ has in $\mathbf{C} \mathbf{L}_{\leftrightarrow}$ the RDP raises the question to what extent it can actually be used as an implication connective. A crucial criterion here is the richness of the languages in which it might serve as such, that is: what useful connectives can be added to it. It can easily be seen that it is impossible to add to $\mathbf{C L}_{\leftrightarrow}$ a 'conjunction' $\wedge$ such that both $\varphi \wedge \psi \leftrightarrow \varphi$ and $\varphi \wedge \psi \leftrightarrow \psi$ would be valid, since this would immediately trivialize the logic. Similarly, one cannot add to $\mathbf{C L}_{\leftrightarrow}$ a 'disjunction' $\vee$ such that $\varphi \leftrightarrow \varphi \vee \psi$ and $\psi \leftrightarrow \varphi \vee \psi$ would be valid. It follows that among the connectives used in linear logic and in relevance logics, one may add to $\mathbf{C L}_{\leftrightarrow}$ only what are called in linear logic 'multiplicative' connectives, and in relevance logics 'intensional' connectives. The most basic such connective is negation (which together with the implication connective of linear and relevant logics suffices for defining the rest of them). Accordingly, the main goal of this paper is to investigate the equivalence-negation fragment of classical logic, and corresponding proof systems.

\section{Preliminaries}

In the sequel $\mathcal{L}$ is a propositional language, $\varphi, \psi, \theta$ vary over its formulas, $p, q$ over its atomic formulas, and $\mathcal{T}, \mathcal{S}$ over its theories (i.e. sets of formulas).

Definition 1. A (Tarskian) consequence relation for a language $\mathcal{L}$ is a binary relation between theories in $\mathcal{L}$ and formulas in $\mathcal{L}$ satisfying the following three conditions:

[R] Reflexivity: $\quad \psi \vdash \psi$ (i.e. $\{\psi\} \vdash \psi$ ).

[M] Monotonicity: $\quad$ if $\mathcal{T} \vdash \psi$ and $\mathcal{T} \subseteq \mathcal{T}^{\prime}$, then $\mathcal{T}^{\prime} \vdash \psi$.

[C] Cut (Transitivity): if $\mathcal{T} \vdash \psi$ and $\mathcal{T}^{\prime}, \psi \vdash \varphi$ then $\mathcal{T} \cup \mathcal{T}^{\prime} \vdash \varphi$.

Definition 2. Let $\vdash$ be a Tarskian consequence relation for $\mathcal{L}$.

- $\vdash$ is structural, if for every $\mathcal{L}$-substitution $\theta$ and every $\mathcal{T}$ and $\psi$, if $\mathcal{T} \vdash \psi$ then $\theta(\mathcal{T}) \vdash \theta(\psi)$.

- $\vdash$ is non-trivial if $p \nvdash q$ for distinct atomic formulas $p, q$.

- $\vdash$ is finitary, if for every theory $\mathcal{T}$ and every formula $\psi$ such that $\mathcal{T} \vdash \psi$ there is a finite theory $\Gamma \subseteq \mathcal{T}$ such that $\Gamma \vdash \psi$. 
Definition 3. A (propositional) logic is a pair $\mathbf{L}=\left\langle\mathcal{L}, \vdash_{\mathbf{L}}\right\rangle$, where $\mathcal{L}$ is a propositional language, and $\vdash_{\mathbf{L}}$ is a structural and non-trivial Tarskian consequence relation for $\mathcal{L}^{2}$ A logic $\mathbf{L}=\left\langle\mathcal{L}, \vdash_{\mathbf{L}}\right\rangle$ is finitary if $\vdash_{\mathbf{L}}$ is finitary.

Definition 4. Let $\mathbf{L}=\left\langle\mathcal{L}, \vdash_{\mathbf{L}}\right\rangle$ be a propositional logic, and let $\supset$ be a (primitive or defined) connective of $\mathcal{L}$. $\mathbf{L}$ has the relevant deduction property (RDP) for $\supset$ if it satisfies the following condition:

$\mathcal{T}, \varphi \vdash_{\mathbf{L}} \psi$ iff either $\mathcal{T} \vdash_{\mathbf{L}} \psi$ or $\mathcal{T} \vdash_{\mathbf{L}} \varphi \supset \psi$

Remark 1. If a finitary logic $\mathbf{L}$ has a connective $\supset$ with the RDP, then the following holds for every theory $\mathcal{T}$ and formula $\varphi: \mathcal{T} \vdash_{\mathbf{L}} \varphi$ iff there exist $\psi_{1}, \ldots, \psi_{n} \in \mathcal{T}(n \geq 0)$ such that $\vdash_{\mathbf{L}} \psi_{1} \supset\left(\psi_{2} \supset\left(\cdots\left(\psi_{n} \supset \varphi\right) \cdots\right)\right)$.

Definition 5. Let $\mathcal{L}_{\rightarrow}=\{\rightarrow\}, \mathcal{L}_{\leftrightarrow}=\{\leftrightarrow\}$.

1. $H L L \rightarrow$ is the system in $\mathcal{L}_{\rightarrow}$ presented in Figure 1

\section{Axioms:}

[Id] $\varphi \rightarrow \varphi$

(Identity)

[Tr] $\quad(\varphi \rightarrow \psi) \rightarrow((\psi \rightarrow \theta) \rightarrow(\varphi \rightarrow \theta))$

(Transitivity)

[Pe] $\quad(\varphi \rightarrow(\psi \rightarrow \theta)) \rightarrow(\psi \rightarrow(\varphi \rightarrow \theta))$

(Permutation)

\section{Rule of inference:}

$[\mathrm{MP}]$

$$
\frac{\varphi \quad \varphi \rightarrow \psi}{\psi}
$$

Fig. 1. The proof system $H L L \rightarrow$

2. $H R_{\rightarrow}$ is the extension of $H L L \rightarrow$ by the following axiom:

$$
\text { [Ct] } \quad(\varphi \rightarrow(\varphi \rightarrow \psi)) \rightarrow(\varphi \rightarrow \psi) \quad \text { (Contraction) }
$$

3. $H L L_{\leftrightarrow}$ is the system in $\mathcal{L}_{\leftrightarrow}$ which is obtained from $H L L \rightarrow$ by using ' $\leftrightarrow$ ' instead of ' $\rightarrow$ '. $H C L_{\leftrightarrow}$ is the extension of $H L L_{\leftrightarrow}$ by the following axiom:

$$
\text { [Eq] } \quad(\varphi \leftrightarrow(\varphi \leftrightarrow \psi)) \leftrightarrow \psi \quad \text { (Equivalence) }
$$

${ }^{2}$ This is the notion of propositional logic which has been used in Avron, 2015, as well as in Avron et al., 2018. 
The following three theorems have been proved in Avron, 2015].

Theorem 1. A logic $\mathbf{L}$ is finitary and has a connective $\rightarrow$ which has in $\mathbf{L}$ the RDP iff $\mathbf{L}$ has a strongly sound and complete Hilbert-type system which is equivalent to an extension by axiom schemas of either $H R_{\rightarrow}$ or $H C L_{\leftrightarrow} \cdot{ }^{3}$

Theorem 2. $H C L_{\leftrightarrow}$ is strongly sound and complete for the equivalence fragment of classical logic (i.e. $\mathcal{T} \vdash_{H C L_{\leftrightarrow}} \varphi$ iff by interpreting $\rightarrow$ as the classical biconditional $\leftrightarrow$, we get that every assignment that satisfies $\mathcal{T}$ also satisfies $\varphi$ ).

Theorem 3. $\mathbf{C L}_{\leftrightarrow}$ has no proper extension in its language. ${ }^{4}$

\section{The Logic $\mathbf{C L}_{\rightleftarrows}$ and the System $H C L_{\rightrightarrows}$}

Definition 6. $\mathbf{C L}_{\rightrightarrows}$ is the equivalence-negation fragment of classical logic. $\mathcal{M}_{C L_{\oiint}}$ is the two-valued matrix which induces $\mathbf{C} \mathbf{L}_{\rightrightarrows}$.

Proposition 1. $\mathrm{CL}_{\rightrightarrows}$ does not have the RDP.

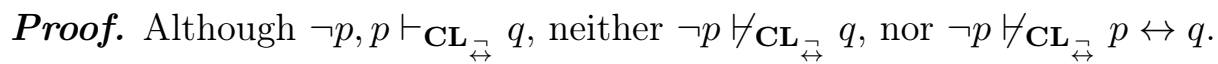

Definition 7. Let $\mathcal{L}=\{\leftrightarrow, \neg\}$. The Hilbert-type proof system $H C L_{\sharp}$ is presented in Figure 2, $\mathbf{L}_{H C L_{\sharp}}$ is the logic induced by $H C L_{\rightrightarrows}$.

Remark 2. The axioms given in Figure 2 are actually not independent, since [N2] can be dropped. This can be seen by substituting $\neg \psi$ for $\varphi$ in both [Id] and [N1]. By applying [MP] to the resulting formulas we get $\psi \leftrightarrow \neg \neg \psi$. From this we can get [N2] by using $H C L_{\leftrightarrow}$ and Theorem $2{ }^{5}$

Remark 3. In Chapter 11 of [Avron et al., 2018] a notion was introduced of a negation associated with a given binary connective that has the RDP. It was shown there that a logic possesses a binary connective $\rightarrow$ that has the RDP together with a negation $\neg$ associated with it iff it is induced by some axiomatic extension of the system $H L L_{\rightrightarrows}$. The latter is the standard Hilbert-type system (given in [Avron, 1988]) for the multiplicative fragment (without the multiplicative constants) of linear logic (|Girard, 1987]). It is obtained from $H C L_{\rightrightarrows}$ by deleting the axiom $[\mathrm{Eq}]$, and then changing $\leftrightarrow$ in all axioms and rules to $\rightarrow$. By adding to $H L L_{\rightrightarrows}$ the contraction axiom, we get the standard Hilbert-type system for $\mathbf{R}_{\rightrightarrows}$, the intensional fragment of the relevance logic $\mathbf{R}$.

\footnotetext{
${ }^{3}$ It seems that the RDP was first raised as being of interest to relevance logic in Diaz, 1980 .

${ }^{4}$ A weaker result, that $\mathbf{C L}_{\leftrightarrow}$ is Post-complete in the sense that one cannot (consistently) add any new axiom to it in its language, had already been shown by Prior in Prior, 1962.

${ }^{5} \mathrm{I}$ am indebted to an anonymous referee for this observation.
} 


\section{Axioms:}

[Id] $\quad \varphi \leftrightarrow \varphi$

(Identity)

[Tr] $(\varphi \leftrightarrow \psi) \leftrightarrow((\psi \leftrightarrow \theta) \leftrightarrow(\varphi \leftrightarrow \theta))$

(Transitivity)

[Pe] $\quad(\varphi \leftrightarrow(\psi \leftrightarrow \theta)) \leftrightarrow(\psi \leftrightarrow(\varphi \leftrightarrow \theta))$

(Permutation)

[Eq] $\quad(\varphi \leftrightarrow(\varphi \leftrightarrow \psi)) \leftrightarrow \psi$

(Equivalence)

[N1] $\quad(\varphi \leftrightarrow \neg \psi) \leftrightarrow(\psi \leftrightarrow \neg \varphi)$

(Contraposition)

[N2] $\neg \neg \varphi \leftrightarrow \varphi$

(Double neg.)

\section{Rule of inference:}

$[\mathrm{MP}]_{\leftrightarrow}$ $\frac{\varphi \quad \varphi \leftrightarrow \psi}{\psi}$

Fig. 2. The proof system $H C L_{\rightrightarrows}$

Theorem 4. Let the logic $\mathbf{L}$ be induced by some axiomatic extension (i.e. extension by axiom schemas) of $\mathrm{HCL}_{\sharp}$. Then $\mathbf{L}$ has the RDP for $\leftrightarrow$.

Proof. Immediate from Theorem 1 .

Theorem 5. $H C L_{\rightrightarrows}$ is strongly sound for $\mathbf{C L}_{\rightrightarrows}$. (I.e., if $\mathcal{T} \vdash_{H C L_{\rightrightarrows}} \varphi$ then by interpreting $\leftrightarrow$ as the classical biconditional $\leftrightarrow$, and $\neg$ as the classical negation, we get that every assignment that satisfies $\mathcal{T}$ also satisfies $\varphi$.)

Proof. Obviously, $[\mathrm{MP}]_{\leftrightarrow}$ is a valid rule of inference for the classical biconditional $\leftrightarrow$. It is also easy to check that every axiom of $H C L_{\rightleftarrows}$ becomes a classical tautology if $\leftrightarrow$ is interpreted as the classical biconditional. Hence $H C L_{\rightleftarrows}$ is strongly sound for the equivalence-negation fragment of classical logic.

Corollary 1. Let $\varphi$ be a formula in the language of $\mathbf{C L}_{\rightleftarrows}$.

1. There is a formula $\psi$ in the language of $\mathbf{C L}_{\leftrightarrow}$ such that:

- If the number of negations in $\varphi$ is even then $\vdash_{\mathbf{C L}_{\rightrightarrows}} \varphi \leftrightarrow \psi$.

- If the number of negations in $\varphi$ is odd then $\vdash_{\mathbf{C L}_{\oiint}} \varphi \leftrightarrow \neg \psi$.

2. $\varphi$ is a classical tautology iff the number of negations in $\varphi$ is even, and for each atomic $p$ the number of occurrences of $p$ in $\varphi$ is even too. ${ }^{6}$

\footnotetext{
${ }^{6}$ According to [Church, 1956, the second item of this Corollary has independently been observed by McKinsey and Mihailescu. As far as I know, the first item was first proved in Mihailescu, 1937.
} 
Proof. Obviously, $H C L_{\sharp}$ has the replacement property. (This is true for every axiomatic extension of $H L L_{\leftrightarrow}$.) It is also easy to show that $\theta_{1} \leftrightarrow \neg \theta_{2}$ and $\neg \theta_{1} \leftrightarrow \theta_{2}$ are both equivalent in $H C L_{\leftrightarrow}$ to $\neg\left(\theta_{1} \leftrightarrow \theta_{2}\right)$. Using these two equivalences and axiom [N2], we can constructively find for $\varphi$ a formula $\psi$ in the language of $\mathbf{C L}_{\leftrightarrow}$ such that $\vdash_{H C L_{\rightleftarrows}} \varphi \leftrightarrow \psi$ or $\vdash_{H C L_{\text {ґ }}} \varphi \leftrightarrow \neg \psi$ according to the parity of $\varphi$ 's number of negations. Hence Theorem 5 implies item 1 .

For the second item, note that if $\psi$ is in the language of $\mathbf{C L}_{\leftrightarrow}$, then $\neg \psi$ is not a tautology, since we can refute it by assigning $t$ to all atomic formulas. Therefore the second part follows from the first, using Leśniewski's famous criterion for being a tautology in $\leftrightarrow$. (See e.g. Corollary 7.31.7 in Humberstone, 2011.)

Theorem 6. No Hilbert-type system which has [MP] for $\leftrightarrow$ as its sole rule of inference can be strongly sound and complete for $\mathbf{C L}_{\rightleftarrows}$.

Proof. Suppose that such a system $H$ exists. From Theorem 5 it follows that we may assume that $H$ is an axiomatic extension of $H C L_{\rightarrow}$. Hence Theorem 1 implies that $H$ has the RDP. Therefore it follows from the strong soundness and completeness of $H$ that so does $\mathbf{C L}_{\rightleftarrows}$, This contradicts Proposition 1 .

Corollary 2. $\mathbf{L}_{H C L_{\rightleftarrows}} \neq \mathbf{C L}_{\rightleftarrows}$, i.e. $H C L_{\rightleftarrows}$ is not strongly complete for $\mathbf{C L}_{\rightleftarrows} \cdot{ }^{7}$

Next we present semantics for $H C L_{\sharp}$ for which this system is strongly sound and complete.

Definition 8. Let $\mathcal{L}=\{\leftrightarrow, \neg\}$. $\mathbf{C L}_{\{\leftrightarrow, i d\}}$ is the two-valued logic which is obtained by interpreting $\leftrightarrow$ as the classical biconditional $\leftrightarrow$, and $\neg$ as the identity connective. $\mathcal{M}_{\mathbf{C L}_{\{\leftrightarrow, i d\}}}$ is the two-valued matrix which induces $\mathbf{C L}_{\{\leftrightarrow, i d\}}$.

For the next proof, we need the following easy lemma, which is directly proved in Avron, 2015 (but also easily follows via an appeal to Lesniewski's criterion, mentioned at the end of the proof of Corollary 1).

\section{Lemma 1.}

$$
\begin{aligned}
& \text { 1. } \vdash_{H C L_{\leftrightarrow}}(\varphi \leftrightarrow \psi) \leftrightarrow(\psi \leftrightarrow \varphi) \\
& \text { 2. } \vdash_{H C L_{\leftrightarrow}} \varphi \leftrightarrow(\psi \leftrightarrow(\varphi \leftrightarrow \psi))
\end{aligned}
$$

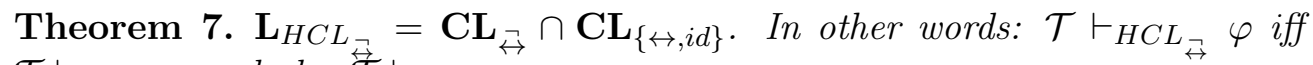
$\mathcal{T} \vdash \mathbf{C L}_{\sharp} \varphi$ and also $\mathscr{\mathcal { T }} \vdash \mathbf{C L}_{\{\leftrightarrow, i d\}} \varphi$.

\footnotetext{
${ }^{7}$ This fact was first noticed in Avron, 2020] (Corollary 14).
} 
Proof. The strong soundness of $H C L_{\rightrightarrows}$ for $\mathbf{C L}_{\{\leftrightarrow, i d\}}$ follows from Theorem 2 . This and Theorem 5 imply the strong soundness of $H C L_{\sharp}$ for $\mathbf{C L}_{\rightrightarrows} \cap \mathbf{C L} \mathbf{L}_{\{\leftrightarrow, i d\}}$. To prove strong completeness, assume that $\mathcal{T} \forall_{H C L_{\sharp}} \theta$. Extend $\stackrel{\leftrightarrow}{\mathcal{T}}$ to a maximal theory $\mathcal{T}^{*}$ such that $\mathcal{T}^{*} \nvdash_{H C L_{\rightrightarrows}} \theta$. Obviously,,$\varphi \in \mathcal{T}^{*}$ iff $\mathcal{T}^{*} \vdash_{H C L_{\rightrightarrows}} \varphi$, and $\varphi \notin \mathcal{T}^{*}$ iff $\mathcal{T}^{*}, \varphi \vdash_{H C L_{\rightleftarrows}} \theta$. Therefore the RDP implies that

$$
\text { (*) } \varphi \notin \mathcal{T}^{*} \quad \text { iff } \quad \varphi \leftrightarrow \theta \in \mathcal{T}^{*}
$$

Now define a valuation $v$ as follows:

$$
v(\varphi)= \begin{cases}t & \text { if } \varphi \in \mathcal{T}^{*} \\ f & \text { if } \varphi \notin \mathcal{T}^{*}\end{cases}
$$

Obviously we have:

(**) $v(\varphi)=t$ for every $\varphi \in \mathcal{T}^{*}$, while $v(\theta)=f$.

Next we show that $v$ respects the truth table of the classical biconditional.

- Suppose $v(\varphi)=v(\psi)=t$. Then $\varphi \in \mathcal{T}^{*}$ and $\psi \in \mathcal{T}^{*}$. Therefore it follows from the second item of Lemma 1 that $\mathcal{T}^{*} \vdash_{H C L_{\text {ङ }}} \varphi \leftrightarrow \psi$. Hence $\varphi \leftrightarrow \psi \in \mathcal{T}^{*}$, and so $v(\varphi \leftrightarrow \psi)=t$.

- Suppose $v(\varphi)=t$ and $v(\psi)=f$. Then $\varphi \in \mathcal{T}^{*}$, while $\psi \notin \mathcal{T}^{*}$. Because of the presence of $[\mathrm{MP}]_{\leftrightarrow}$, these facts immediately imply that $\varphi \leftrightarrow \psi \notin \mathcal{T}^{*}$, and so $v(\varphi \leftrightarrow \psi)=f$ in this case.

- Suppose $v(\varphi)=f$ and $v(\psi)=t$. By the previous item this implies that $\psi \leftrightarrow \varphi \notin \mathcal{T}^{*}$. Therefore the first item of Lemma 11 implies that $\varphi \leftrightarrow \psi \notin \mathcal{T}^{*}$, and so $v(\varphi \leftrightarrow \psi)=f$ in this case too.

- Suppose $v(\varphi)=v(\psi)=f$. Then $\varphi \notin \mathcal{T}^{*}$ and $\psi \notin \mathcal{T}^{*}$. By $\left(^{*}\right)$ above, it follows that $\varphi \leftrightarrow \theta \in \mathcal{T}^{*}$ and $\psi \leftrightarrow \theta \in \mathcal{T}^{*}$. By the first item of Lemma 1 , the second fact implies that $\theta \leftrightarrow \psi \in \mathcal{T}^{*}$. Using [Tr] this last fact and the fact that $\varphi \leftrightarrow \theta \in \mathcal{T}^{*}$ together imply that $\varphi \leftrightarrow \psi \in \mathcal{T}^{*}$, and so $v(\varphi \leftrightarrow \psi)=t$ in this case.

To determine the behavior of $v$ with respect to $\neg$ we have two cases to consider. $\neg \theta \in \mathcal{T}^{*}$

- Suppose $v(\varphi)=t$. Then $\varphi \in \mathcal{T}^{*}$. By the second item of Lemma 1 . this implies in this case that $\varphi \leftrightarrow \neg \theta \in \mathcal{T}^{*}$. It follows by [N1] that $\theta \leftrightarrow \neg \varphi \in \mathcal{T}^{*}$, and so $\neg \varphi \leftrightarrow \theta \in \mathcal{T}^{*}$ by the first item of Lemma 1 . Hence $\left(^{*}\right)$ entails that $\neg \varphi \notin \mathcal{T}^{*}$, implying that $v(\neg \varphi)=f$ in this case. 
- Suppose $v(\varphi)=f$. Then $\varphi \notin \mathcal{T}^{*}$. Hence $\left(^{*}\right)$ entails that $\varphi \leftrightarrow$ $\theta \in \mathcal{T}^{*}$. By [N1] and [N2], this implies that $\neg \theta \leftrightarrow \neg \varphi \in \mathcal{T}^{*}$, and so $\neg \varphi \in \mathcal{T}^{*}$ (since we are assuming that $\neg \theta \in \mathcal{T}^{*}$ ). Therefore $v(\neg \varphi)=t$ in this case.

It follows that $v$ is a legal valuation of the classical equivalence-negation matrix $\mathcal{M}_{C L_{\sharp}}$. Hence $\mathcal{T} \nvdash_{\mathbf{C L}_{\sharp}} \theta$ in this case.

$\neg \theta \notin \mathcal{T}^{*}$

- Suppose $v(\varphi)=t$. Then $\varphi \in \mathcal{T}^{*}$. Suppose that $\neg \varphi \notin \mathcal{T}^{*}$. Then by $\left(^{*}\right), \neg \varphi \leftrightarrow \theta \in \mathcal{T}^{*}$, and so the first item of Lemma 1 implies that $\theta \leftrightarrow \neg \varphi \in \mathcal{T}^{*}$. Hence [N1] entails that $\varphi \leftrightarrow \neg \theta \in \mathcal{T}^{*}$. Since $\varphi \in \mathcal{T}^{*}$, $\neg \theta \in \mathcal{T}^{*}$ too. A contradiction. It follows that $\neg \varphi \in \mathcal{T}^{*}$, and so $v(\neg \varphi)=t$.

- Suppose $v(\varphi)=f$. Then $\varphi \notin \mathcal{T}^{*}$, implying by $\left({ }^{*}\right)$ that $\varphi \leftrightarrow \theta \in \mathcal{T}^{*}$. Hence the first item of Lemma 1 implies that $\theta \leftrightarrow \varphi \in \mathcal{T}^{*}$. Using [N1], [N2], and Lemma 1, we get from this that $\neg \varphi \leftrightarrow \neg \theta \in \mathcal{T}^{*}$. Since $\neg \theta \notin \mathcal{T}^{*}$, this means that $\neg \varphi \notin \mathcal{T}^{*}$ too, and so $v(\neg \varphi)=f$.

We have shown that $v$ is a legal valuation of the classical equivalenceidentity matrix $\mathcal{M}_{\mathbf{C L}_{\{\leftrightarrow, i d\}}}$. Hence $\mathcal{T}^{*} \nvdash_{\mathbf{C L}_{\{\leftrightarrow, i d\}}} \theta$ in this case.

It follows that if $\mathcal{T} \nvdash_{H C L_{\rightleftarrows}} \theta$ then either $\mathcal{T} \nvdash_{\mathbf{C L}_{\rightleftarrows}} \theta$ or $\mathcal{T} \nvdash_{\mathbf{C L}_{\{\leftrightarrow, i d\}}} \theta$.

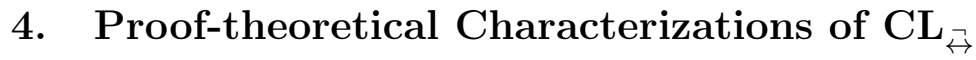

Theorem 8. $\mathcal{T} \vdash_{\mathrm{CL}_{\oiint}} \varphi$ iff $\mathcal{T}, \neg \varphi \vdash_{H C L_{\rightleftarrows}} \varphi$.

Proof. Suppose $\mathcal{T} \vdash_{\mathbf{C L}_{\sharp}} \varphi$. Then $\mathcal{T}, \neg \varphi \vdash_{\mathbf{C L}_{\sharp}} \varphi$. Obviously, $\mathcal{T}, \neg \varphi \vdash_{\mathbf{C L}_{\{\leftrightarrow, i d\}}}$ $\varphi$ as well. Hence Theorem 7 implies that $\mathcal{T}, \neg \stackrel{\leftrightarrow}{\varphi} \vdash_{H C L_{\text {ङ }}} \varphi$.

For the converse, let $\mathcal{T}, \neg \varphi \vdash_{H C L_{\rightrightarrows}} \varphi$. Suppose that $\mathcal{T} \forall_{\mathbf{C L}_{\sharp}} \varphi$. Then there is a a valuation $v$ in $\mathcal{M}_{C L_{\rightleftarrows}}$ such that $v(\psi)=t$ for every $\psi \in \mathcal{T}$, while $v(\varphi)=f$. The latter fact implies that $v(\neg \varphi)=t$, and so $v$ is a model in $\mathcal{M}_{C L_{\rightleftarrows}}$ of $\mathcal{T} \cup\{\neg \varphi\}$ which is not a model in $\mathcal{M}_{C L_{\rightrightarrows}}$ of $\varphi$. Since $\mathcal{T}, \neg \varphi \vdash_{H C L_{\rightrightarrows}} \varphi$, this contradicts Theorem 5 .

Theorem 9. $H C L_{\rightrightarrows}$ is weakly complete for $\mathbf{C L}_{\rightrightarrows}: \vdash_{H C L_{\oiint}} \varphi$ iff $\vdash_{\mathbf{C L}_{\sharp}} \varphi$.

Proof. Theorem 5 implies the 'only if' part. For the converse, let $\vdash \mathbf{C L}_{\rightrightarrows} \varphi$. By Theorem 8, it follows that $\neg \varphi \vdash_{H C L_{\rightrightarrows}} \varphi$. Hence Theorem 4 implies that either $\vdash_{H C L_{\rightleftarrows}} \neg \varphi \leftrightarrow \varphi$, or $\vdash_{H C L_{\rightleftarrows}} \varphi$. The first option is impossible by Theorem 5 since $\stackrel{\leftrightarrow}{v}(\neg \varphi \leftrightarrow \varphi)=f$ for every valuation $v$ in $\mathcal{M}_{C L_{\sharp}}$. Hence $\vdash_{H C L_{\sharp}} \varphi$. 
Remark 4. Theorem 9 easily follows also from Corollary 1 and Theorem $2{ }^{8}$

Theorem 10. Let $H C L_{\rightrightarrows}^{\star}$ be obtained from $H C L_{\rightrightarrows}$ by adding to it the Ex Falso rule $\frac{\neg \varphi \varphi}{\psi}$ as a rule of inference. Then $H C L_{\leftrightarrow}^{\star}$ is strongly sound and complete for $\mathbf{C L}_{\rightleftarrows}$.

Proof. That $H C L_{\sharp}^{\star}$ is strongly sound for $\mathbf{C L}_{\rightleftarrows}$ follows from Theorem 5 , and the strong soundness in classical logic of the special rule of $H C L_{\stackrel{\leftrightarrow}{\star}}^{\star}$.

To show strong completeness, let $\mathcal{T} \vdash_{\mathbf{C L}_{\rightrightarrows}} \varphi$. Then $\mathcal{T}, \neg \varphi \stackrel{\leftrightarrow}{\vdash}_{H C L_{\sharp}} \varphi$ by

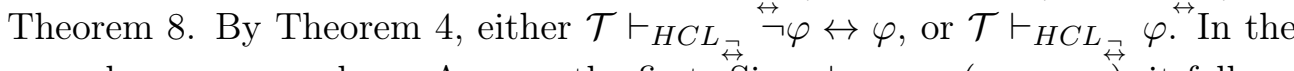
second case we are done. Assume the first. Since $\vdash_{\mathbf{C L}_{\sharp}} \neg(\neg \varphi \leftrightarrow \varphi)$, it follows by Theorem 9 that $\mathcal{T} \vdash_{H C L_{\sqsupset}} \neg(\neg \varphi \leftrightarrow \varphi)$ as well. Hence an application of the Ex Falso rule of $H C L_{\rightleftarrows}^{\star}$ yields that $\mathcal{T}^{\star} \vdash_{\mathbf{C L}_{\rightleftarrows}} \varphi$.

\section{Corollary 3.}

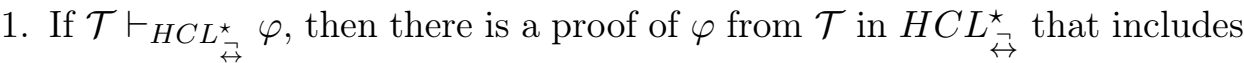
at most one application, made at the end of that proof, of its extra rule.

2. $\mathcal{T} \vdash_{\mathbf{C L}_{\sharp}} \varphi$ iff either $\mathcal{T} \vdash_{H C L_{\rightrightarrows}} \varphi$ or $\mathcal{T}$ is inconsistent in $H C L_{\rightleftarrows}$.

Proof. The first part easily follows from the proof of Theorem 10. The second one follows from that theorem together with the first part.

Turning to a corresponding Gentzen-type System, we note that in Avron, Lev, 2005 an algorithm has been given for finding a cut-free sound and complete Gentzen-type system for every logic which has a two-valued characteristic matrix (or even non-deterministic matrix). By applying that algorithm to $\mathbf{C L}_{\rightleftarrows}$, we get the system $G C L_{\rightrightarrows}$ presented at Figure 3 . In this presentation $\Gamma$ and $\stackrel{\leftrightarrow}{\Delta}$ vary over finite sets of formulas.

\section{Theorem 11.}

1. $G C L_{\rightrightarrows}$ is strongly sound and complete for $\mathbf{C L}_{\rightrightarrows}$.

2. The cut-elimination theorem obtains for $G C L_{\rightleftarrows}:$ if $\vdash_{G C L_{\sharp}} \Gamma \Rightarrow \Delta$, then $\Gamma \Rightarrow \Delta$ has a cut-free proof in $G C L_{\rightleftarrows}$.

Proof. This is a special case of Theorem 4.7 of Avron, Lev, 2005 and its proof.

\footnotetext{
${ }^{8}$ Theorem 9 was essentially first proved in Mihailescu, 1937. (See also Bennett, 1937.)
} The system used there is easily seen to be equivalent to $H C L_{\rightleftarrows}$. 
Axioms: $\varphi \Rightarrow \varphi$

Rules: cut, weakening, and the following logical rules:

$$
\begin{array}{ll}
\frac{\Gamma, \varphi, \psi \Rightarrow \Delta \quad \Gamma \Rightarrow \Delta, \varphi, \psi}{\Gamma, \varphi \leftrightarrow \psi \Rightarrow \Delta} & \frac{\Gamma, \psi \Rightarrow \Delta, \varphi}{\Gamma \Rightarrow \Delta, \varphi \leftrightarrow \psi} \quad \Gamma, \varphi \Rightarrow \Delta, \psi \\
\frac{\Gamma \Rightarrow \Delta, \varphi}{\Gamma, \neg \varphi \Rightarrow \Delta} & \frac{\Gamma, \varphi \Rightarrow \Delta}{\Gamma \Rightarrow \Delta, \neg \varphi}
\end{array}
$$

Fig. 3. $G C L_{\text {马 }}$

Remark 5. A model of a sequent $\Gamma \Rightarrow \Delta$ in a two-valued matrix $\mathcal{M}$ is usually taken to be a valuation $v$ in $\mathcal{M}$ such that $v(\varphi)=f$ for some $\varphi \in \Gamma$, or $v(\varphi)=t$ for some $\varphi \in \Delta$. Let $S \cup\{s\}$ be a set of sequents. Define: $S \vdash_{\mathcal{M}} s$ if every model of $S$ in $\mathcal{M}$ is also a model of $s$. The first item of Theorem 11 means that $S \vdash \mathrm{CL}_{\rightleftarrows} s$ iff $S \vdash_{G C L_{\rightleftarrows}} s$.

\section{The Expressive Power of $\mathrm{CL}_{\rightrightarrows}$}

To make our treatment of $\mathbf{C L}_{\rightrightarrows}$ complete, we include also a characterization of the set of two-valued connectives that are definable in the language of $\mathbf{C L}_{\rightleftarrows}$. For this it would be convenient to use 1 and -1 as our truth-values.

Definition 9. Let $H:\{1,-1\}^{n} \rightarrow\{1,-1\}$, and let $x_{1}, \ldots, x_{n}$ be $n$ variables.

- For $1 \leq i \leq n, x_{i}$ is a dummy variable of $H\left(x_{1}, \ldots, x_{n}\right)$ if

$$
H\left(x_{1}, \ldots, x_{i-1},-x_{i}, x_{i+1}, \ldots, x_{n}\right)=H\left(x_{1}, \ldots, x_{i-1}, x_{i}, x_{i+1}, \ldots, x_{n}\right)
$$

for every $x_{1}, x_{2}, \ldots, x_{n} \in\{-1,1\}$.

- For $1 \leq i \leq n, x_{i}$ is a flipping variable of $H\left(x_{1}, \ldots, x_{n}\right)$ if

$$
H\left(x_{1}, \ldots, x_{i-1},-x_{i}, x_{i+1}, \ldots, x_{n}\right)=-H\left(x_{1}, \ldots, x_{i-1}, x_{i}, x_{i+1}, \ldots, x_{n}\right)
$$

for every $x_{1}, x_{2}, \ldots, x_{n} \in\{-1,1\}$.

Theorem 12. A function $H:\{1,-1\}^{n} \rightarrow\{1,-1\}$ is definable by a formula of $\mathbf{C L}_{\rightleftarrows}$ iff for every $1 \leq i \leq n, x_{i}$ is either a dummy variable or a flipping variable of $H\left(x_{1}, \ldots, x_{n}\right) .^{9}$

\footnotetext{
${ }^{9}$ This theorem can most probably be extracted from Post's discussion in Post, 1941.
} 
Proof. That any function which is definable by a formula $\varphi$ of $\mathbf{C L}_{\rightleftarrows}$ satisfies the condition is easily proved by induction on the structure of $\varphi$.

For the converse, suppose $H:\{1,-1\}^{n} \rightarrow\{1,-1\}$ satisfies the condition. Let $\psi=\psi_{1} \leftrightarrow \cdots \leftrightarrow \psi_{n}$, where $\psi_{i}=p_{i} \leftrightarrow p_{i}$ if $x_{i}$ is a dummy variable of $H\left(x_{1}, \ldots, x_{n}\right), \psi_{i}=p_{i}$ if $x_{i}$ is a flipping variable of $H\left(x_{1}, \ldots, x_{n}\right)$. It is not difficult to see that $\psi$ defines $H$ in case $H(1,1, \ldots, 1)=1$, while $\neg \psi$ defines $H$ in case $H(1,1, \ldots, 1)=-1$.

Corollary 4. The classical conjunction, disjunction, and (material) implication are not definable by a formula of $\mathbf{C L}_{\rightleftarrows} \cdot{ }^{10}$

\section{Extensions of $\mathbf{L}_{H C L_{\text {马 }}}$}

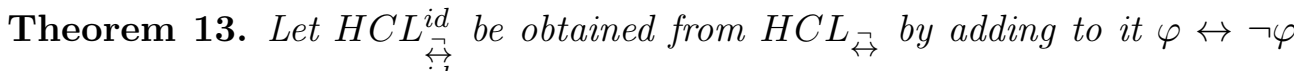
as an axiom. Then $H C L_{\leftrightarrow}^{\stackrel{i d}{\leftrightarrow}}$ is strongly sound and complete for $\mathbf{C L}_{\{\leftrightarrow, i d\}}$.

Proof. The proof is almost identical to that of Theorem 7. We only need to observe that in the presence of the additional axiom, the case in which $\neg \theta \in \mathcal{T}^{*}$ is impossible. Hence we remain with the case in which $\mathcal{T}^{*} \nvdash_{\mathbf{C L}_{\{\leftrightarrow, i d\}}} \theta$.

Theorem 14. $\mathbf{C L}_{\oiint}$ and $\mathbf{C L}_{\{\leftrightarrow, i d\}}$ are the sole non-trivial proper extensions of $\mathbf{L}_{H C L_{\rightleftarrows}}$ in its language.

Proof. Let $\mathbf{L}$ be a logic in the language of $H C L_{\rightrightarrows}$ which is a proper extension of $\mathbf{L}_{H C L_{\Im}}$. Then there is a theory $\mathcal{T}$ and a formula $\theta$ such that $\mathcal{T} \vdash_{\mathbf{L}} \theta$, but $\mathcal{T} H H_{H C L_{\rightrightarrows}} \theta$. By Theorem 7 there is an assignment $v$ in either $\mathcal{M}_{C L_{\rightrightarrows}}$ or $\mathcal{M}_{\mathbf{C L}_{\{\leftrightarrow, i d\}}}$ such that $v(\varphi)=t$ for every $\varphi \in \mathcal{T}$, while $v(\theta)=f$. Pick some atomic formula $p$, and define a substitution $S$ by:

$$
S(q)= \begin{cases}p \leftrightarrow p & \text { if } v(q)=t \\ p & \text { if } v(q)=f\end{cases}
$$

Let $\mathcal{T}^{\star}=\{S(\varphi) \mid \varphi \in \mathcal{T}\}, \theta^{\star}=S(\theta)$. Since $\mathbf{L}$ is a logic, $\mathcal{T}^{\star} \vdash_{\mathbf{L}} \theta^{\star}$. On the other hand, the obvious fact that $v(S(\varphi))=v(\varphi)$ for every formula $\varphi$ implies that $v=\mathcal{T}^{\star}$, while $v \not \models \theta^{\star}$. It follows by Theorem 7 that $\mathcal{T}^{\star} \nvdash_{H C L_{\rightleftarrows}} \theta^{\star}$.

Now from Theorem 9 it follows (and it can also easily be shown directly) that every formula which has $p$ as its sole atomic subformula is equivalent in $H C L_{\rightleftarrows}$ to one of the formulas in $\{p, \neg p, p \leftrightarrow p, \neg(p \leftrightarrow p)\}$. Hence $\mathcal{T}^{\star} \cup\left\{\theta^{\star}\right\}$ is a subset of this set. Moreover, the fact that $\vdash_{H C L_{\rightleftarrows}} p \leftrightarrow p$ implies that $\theta^{\star} \neq p \leftrightarrow p$, and

\footnotetext{
${ }^{10}$ This result has been shown directly in Massey, 1977. It is also proved there that there is no single truth-function that generates precisely the functions definable by formulas of $\mathbf{C L}_{\rightleftarrows}$.
} 
that we may assume that $p \leftrightarrow p \notin \mathcal{T}^{\star}$. Hence $\mathcal{T}^{\star} \cup\left\{\theta^{\star}\right\} \subseteq\{p, \neg p, \neg(p \leftrightarrow p)\}$. However, since $\vdash_{H C L_{\rightleftarrows}} p \leftrightarrow \neg p \leftrightarrow \neg(p \leftrightarrow p)$ by Theorem 9, any element of $\{p, \neg p, \neg(p \leftrightarrow p)\}$ follows in $H C L_{\rightrightarrows}$ from the other two. Therefore we remain with the following six cases.

$p \vdash_{\mathbf{L}} \neg(p \leftrightarrow p)$ : Substituting $p \leftrightarrow p$ for $p$, we get that $\vdash_{\mathbf{L}} \neg((p \leftrightarrow p) \leftrightarrow(p \leftrightarrow p)$. But by Theorem 9, $\neg\left((p \leftrightarrow p) \leftrightarrow(p \leftrightarrow p)\right.$ is equivalent in $H C L_{\rightleftarrows}$ to $\neg p \leftrightarrow p$, and so $\vdash_{\mathbf{L}} \neg p \leftrightarrow p$. Hence $\mathbf{L}$ extends $\mathbf{C L}_{\{\leftrightarrow, i d\}}$.

$p \vdash_{\mathbf{L}} \neg p$ : Substituting $p \leftrightarrow p$ for $p$, we again get that $\mathbf{C L}_{\{\leftrightarrow, i d\}} \subseteq \mathbf{L}$.

$\neg p \vdash_{\mathbf{L}} \neg(p \leftrightarrow p)$ : Substituting $\neg(p \leftrightarrow p)$ for $p$, we again get that $\mathbf{C L}_{\{\leftrightarrow, i d\}} \subseteq \mathbf{L}$.

$\neg p \vdash_{\mathbf{L}} p$ : Substituting $\neg(p \leftrightarrow p)$ for $p$, we again get that $\mathbf{C L}_{\{\leftrightarrow, i d\}} \subseteq \mathbf{L}$.

$\neg(p \leftrightarrow p) \vdash_{\mathbf{L}} p$ : Since $\vdash_{H C L_{\rightrightarrows}} q \leftrightarrow \neg q \leftrightarrow(\neg(p \leftrightarrow p))$ by Theorem 9 , we get that $q, \neg q \vdash_{\mathbf{L}} p$. Hence $\mathbf{L}$ extends $\mathbf{C L}_{\leftrightarrow}$ in this case (by Theorem 10 .

$\neg(p \leftrightarrow p) \vdash_{\mathbf{L}} \neg p$ : Here we get by a similar argument that $q, \neg q \vdash_{\mathbf{L}} \neg p$. Substituting $\neg p$ for $p$, it follows (using [N2]) that $q, \neg q \vdash_{\mathbf{L}} p$. Hence $\mathbf{L}$ extends $\mathbf{C L}_{\rightleftarrows}$ in this case too.

We have shown that $\mathbf{L}$ extends either $\mathbf{C} \mathbf{L}_{\rightleftarrows}$ or $\mathbf{C} \mathbf{L}_{\{\leftrightarrow, i d\}}$. However, since these are two-valued logics, neither of them has a proper non-trivial extension, by a general theorem in Rautenberg, 1981]. (These two cases can also be shown directly using an analysis which is very similar to - though shorter and easier than - that given above for $\mathbf{L}_{H C L_{\sharp}}$.) Hence $\mathbf{L}$ is necessarily one of them.

Corollary 5. $H C L_{\leftrightarrow}^{i d}$ is the sole non-trivial proper axiomatic extension of $H C L_{\rightleftarrows} \cdot{ }^{11}$

Remark 6. Recall that theorems 2 and 3 imply Prior's result that $H C L_{\leftrightarrow}$ is Post-complete (footnote 4). Corollary 5 means that in contrast, $H C L_{\rightleftarrows}$ is not Post-complete. ${ }^{12}$ However, the difference from $H C L_{\leftrightarrow}$ is small: $H C \overleftrightarrow{L}_{\rightleftarrows}$ has just one proper axiomatic extension.

\section{Is $\mathbf{L}_{H C L_{\sharp}}$ a Paraconsistent Logic?}

In Chapter 2 of Avron et al., 2018] a propositional $\mathbf{L}$ for a language with a unary connective $\neg$ is defined to be $\neg$-paraconsistent if $p, \neg p \nvdash_{\mathbf{L}} q$ whenever $p$ and $q$ are distinct variables, and $\neg$ is a negation of $\mathbf{L} . \mathbf{L}_{H C L_{\sharp}}$ certainly satisfies

\footnotetext{
${ }^{11}$ This fact was first proved in Avron, 2020 (Theorem 16).

${ }^{12}$ That $\mathbf{C L}_{\rightleftarrows}$ has no Post-complete axiomatization has already been noted in Prior, 1962.
} 
the first condition. ${ }^{13}$ Hence the question whether it is $\neg$-paraconsistent depends on whether its connective $\neg$ can be viewed as a negation. This, in turn, depends of course on the definition of negation that one adopts.

In the literature one can find many different definitions of "negation" in $\mathbf{L}$. Some make very weak demands. The minimal ones might be that if $p$ is atomic then $p \nvdash_{\mathbf{L}} \neg p$ and $\neg p \nvdash_{\mathbf{L}} p$. A more extensive set of negative conditions of this sort (divided into two groups, called 'verificatio' and 'falsificatio') is given in Marcos, 2005. It is also possible to add some positive conditions, like that $p \vdash_{\mathbf{L}} \neg \neg p$ and $\neg \neg p \vdash_{\mathbf{L}} p$. All these conditions are satisfied in $\mathbf{L}_{H C L_{\rightleftarrows}}$. So according to weak definitions of this sort, $\mathbf{L}_{H C L_{\rightrightarrows}}$ is indeed a $\neg$-paraconsistent logic.

In Avron et al., 2018, a more restrictive definition of negation has been used. $\neg$ is called there a negation for $\mathbf{L}$ if it is possible to define in the language of $\mathbf{L}$ a binary connective $\diamond$ which is either a disjunction for $\mathbf{L}$, or a conjunction for $\mathbf{L}$, or a semi-implication for $\mathbf{L}$, such that the $\{\neg, \diamond\}$-fragment of $\mathbf{L}$ is contained in the corresponding fragment of classical logic. In order to see that no such connective $\diamond$ is available in $\mathbf{L}_{H C L_{\sqsupset}}$, we do not need to repeat the definitions given in Chapter 1 of Avron et al., 2018] of these notions. It suffices to recall the following facts about them. (They all easily follow from the definitions.)

- If a connective $\wedge$ is a conjunction for a logic $\mathbf{L}$, then for every $\varphi$ and $\psi: \varphi \wedge \psi \vdash_{\mathbf{L}} \varphi, \varphi \wedge \psi \vdash_{\mathbf{L}} \psi$, and $\varphi \vdash_{\mathbf{L}} \varphi \wedge \varphi$. From Theorem 7 it easily follows that such a connective should have in $\mathcal{M}_{C L_{\Im}}$ the truth-table of the classical conjunction. Hence Corollary 4 implies that no such connective is available in $\mathbf{L}_{H C L_{\rightleftarrows}}$.

- If a connective $V$ is a disjunction for a logic $\mathbf{L}$, then for every $\varphi$ and $\psi: \varphi \vdash_{\mathbf{L}} \varphi \vee \psi, \psi \vdash_{\mathbf{L}} \varphi \vee \psi$, and $\varphi \vee \varphi \vdash_{\mathbf{L}} \varphi$. From Theorem 7 it easily

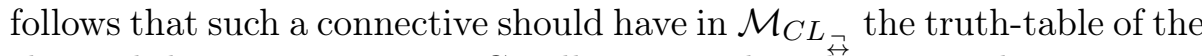
classical disjunction. Hence Corollary 4 implies that no such connective is available in $\mathbf{L}_{H C L_{\sharp}}$.

- If a connective $\supset$ is a semi-implication for a logic $\mathbf{L}$, then it has in $\mathbf{L}$ the RDP. Suppose now that $\supset$ is such a connective in $\mathbf{L}_{H C L_{\sharp}}$. Then $\vdash_{H C L_{\rightleftarrows}} p \supset p$ for every atomic $p$. Hence $\vdash_{H C L_{\rightrightarrows}}(p \supset q) \supset(p \supset q)$ (by structurality). Therefore two applications of the RDP for $\supset$ yield $p, p \supset q \vdash_{H C L_{\sharp}} q$. By two other applications of the RDP, this time for $\leftrightarrow$,

\footnotetext{
${ }^{13}$ In fact, $\mathbf{L}_{H C L_{Z}}$ satisfies the stronger condition $p, \neg p \nvdash_{\mathbf{L}} \neg q$. So if we accept its connective $\neg$ as a negation, then according to Avron et al., 2018 it would even be strongly $\neg$-paraconsistent.
} 
we get that $\vdash_{H C L_{\rightleftarrows}}(p \supset q) \leftrightarrow(p \leftrightarrow q)$. It follows that $\varphi \supset \psi$ and $\varphi \leftrightarrow \psi$ are equivalent in ${\stackrel{\mathbf{L}}{H C L_{\oiint}}}_{\text {. }}$ Therefore $\vdash_{H C L_{\rightleftarrows}}(p \supset(p \supset q)) \supset q$. Hence the $\{\neg, \supset\}$-fragment of $\mathbf{L}_{H C L_{\sharp}}$ is not contained in the corresponding fragment of classical logic.

It follows from the above considerations that $\mathbf{L}_{H C L_{3}}$ is not a paraconsistent logic according to the definition used in Avron et al., 2018].

Acknowledgements. I am very grateful to Lloyd Humberstone for many most helpful comments, suggestions, and pointers to the literature. This research was supported by The Israel Science Foundation (grant no. 817-15).

\section{References}

Anderson, Belnap, 1975 - Anderson, A.R., Belnap, N.D. Entailment: The Logic of Relevance and Necessity. Vol. I. Princeton University Press, 1975.

Avron, 1988 - Avron, A. "The Semantics and Proof Theory of Linear Logic", Theoretical Computer Science, 1988, Vol. 57, pp. 161-184.

Avron, 2015 - Avron, A. "Semi-implication: A chapter in universal logic", The Road to Universal Logic, Volume I., ed. by A. Koslow and A. Buchsbaum. Studies in Universal Logic, Birkhäuser, 2015, pp. 59-72.

Avron, 2020 - Avron, A. "A Note on Semi-implication with Negation", Abstract Consequence and Logics: Essays in Honor of Edelcio G. de Souza, ed. by A. CostaLeite. Tributes 42, College Publications, 2020, pp. 221-226.

Avron et al., 2018 - Avron, A., Arieli, O., Zamansky, A. Theory of Effective Propositional Paraconsistent Logics. volume 75 of Studies in Logic, (Mathematical Logic and Foundations), College Publications, 2018.

Avron, Lev, 2005 - Avron, A., Lev, I. "Non-Deterministic Multiple-valued Structures", Journal of Logic and Computation, 2005, Vol. 15, pp. 241-261.

Bennett, 1937 - Bennett, A.A. Review of [Mihailescu, 1937], Journal of Symbolic Logic, 1937, Vol. 2, No. 4, p. 173.

Church, 1956 - Church, A. Introduction to Mathematical Logic. Princeton University Press, 1956.

Diaz, 1980 - Diaz, M.R. "Deductive Completeness and Conditionalization in Systems of Weak Implication", Notre Dame Journal of Formal Logic, 1980, Vol. 21, pp. 119-130.

Dunn, Restall, 2002 - Dunn, J.M., Restall, G. "Relevance logic", Handbook of Philosophical Logic, Second edition, Vol. 6, ed. by D. Gabbay and F. Guenthner. Kluwer, 2002 pp. 1-136.

Girard, 1987 - Girard, J.Y. "Linear logic", Theoretical Computer Science, 1987, Vol. 50, pp. 1-102.

Humberstone, 2011 - Humberstone, L. The Connectives. The MIT Press, 2011.

Marcos, 2005 - Marcos, J. "On negation: Pure local rules", Journal of Applied Logic, 2005, Vol. 3, pp. 185-219. 
Massey, 1977 - Massey, G.J. "Negation, Material Equivalence, and Conditioned Nonconjunction: Completeness and Duality", Notre Dame Journal of Formal Logic, 1977, Vol. 18, pp. 140-144.

Mihailescu, 1937 - Mihailescu, E.Gh. "Recherches sur la negation el l'équivalence dans le calcul des proposition", Annales scientifiques de l'Université de Jassy, 1937, Vol. 23, pp. 369-408.

Post, 1941 - Post, E. The Two-Valued Iterative Systems of Mathematical Logic. Annals of Mathematics Series, Vol. 5, Princeton University Press, Princeton, NJ, 1941.

Prior, 1962 - Prior, A.N. Formal Logic, second edition. Oxford, 1962.

Rautenberg, 1981 - Rautenberg, W. "2-Element Matrices", Studia Logica, 1981, Vol. 40, pp. 315-353. 